\title{
The Italian reference site for TEM methods
}

\author{
Vincenzo Sapia ${ }^{1,}$, Andrea Viezzoli ${ }^{2}$, Antonio Menghini ${ }^{2}$, Marco Marchetti $^{1}$, Massimo Chiappini $^{1}$ \\ ${ }^{1}$ Istituto Nazionale di Geofisica e Vulcanologia, Rome, Italy \\ ${ }^{2}$ Aarhus Geophysics Aps, Aarhus, Denmark
}

\author{
Article history \\ Received June 10, 2015; accepted August 4, 2015. \\ Subject classification: \\ Time-domain electromagnetic method, Data calibration, Reference site, $1 \mathrm{D}$ resistivity model.
}

\begin{abstract}
The success of a long term transient electromagnetic survey (TEM) rigorously calls for appropriate system calibration, in addition to advanced processing and inversion of the measured data. In fact, acquisition of TEM data can be affected by a variety of noise sources from both inside and outside the system, making it difficult, for example, to define an absolute turn off time and/or to synchronize transmitter and receiver. For these reasons, a reference site plays an important role. As first step, we performed the calibration of a Geonics 47 at the Lyngby reference site in Denmark. We then set up a new reference site using the same calibrated TEM instrument. The reference site was established in the San Rossore park area (Pisa), where we identified an area that matches the required conditions. Subsequently, a series of TEM measurements were collected in the selected area using two pre-calibrated TEM instruments: the Geonics 47 and the WalkTEM respectively. The reference responses were therefore jointly inverted, obtaining a 5 layers model that was appointed to be the TEM reference model for the site. Afterwards, based on that reference model, we calibrated the Geonics 47 and 57 instruments for a $100 \times 100$ $m$ central loop configuration. A unique time-shift and a data level shift factor was calculated and applied to the TEM system as result of the calibration procedure. The San Rossore TEM reference site is now available for anyone interested in calibrating TEM systems.
\end{abstract}

\section{Introduction}

Near surface resolution of transient electromagnetic (TEM) is highly dependent on the specific instrument's capability to measure the early time portion of the very shallow portion (i.e. tens of meters) of the transient ground response. However, acquisition of TEM data can be impaired by a variety of noise sources from both internal and external effects, that mainly affects this part of the measured transient curve. In addition, it is also duly demonstrated that TEM data can suffer from issues due to the inherent difficulty in the description of the key-parameters of the instrumentation like an absolute turn off time and/or the transmitter and receiver synchronization [Christiansen et al.
2011]. All these system-specific inaccuracies directly turn out to inappropriate 1D modeling of the subsoil, both in the very shallow as well as in the deeper part of the transient response.

With regards to near surface resolution, the receiver bandwidth and the current waveform significantly influence the resulting near-surface resolution for any TEM systems. In fact, each TEM system, both ground and airborne, adopts a specific waveform at one or more repetition frequencies in order to fulfill precise objectives and strategies [Liu 1998].

The success of a TEM survey (but also of any geophysical surveys) rigorously calls for appropriate system calibration in addition to advanced processing and inversion of the measured data.

It has been increasingly demonstrated in the recent literature the importance to have calibrated instruments to derive reliable geophysical models for geo $\backslash$ hydrogeological interpretation from TEM data, both from ground and helicopter-borne (HTEM) measurements [Nielsen et al. 2007, Santilano et al. 2015]. An alternative approach might be also the use of a calibrated ground based TEM system to check and post-calibrate an HTEM dataset [i.e. Podgorski et al. 2013].

As demonstrated by the Danish experience, the purpose of the test site is to check that all the TEM systems applied for hydrogeological prospections, are capable of identifying the test site reference model before getting through the specific survey. This is particularly crucial for hydrogeological investigations where the electromagnetic signatures are lower than those of mineral exploration targets [Foged et al. 2013]. The Danish TEM test site is located at Lyngby near Aarhus (Denmark) and was set up in 2001. Over the years, it has been used to calibrate TEM instruments from Denmark as well as France, Germany, Switzerland, Australia, United States. 
Originally the test site adopted a single TEM sounding with a unique derived reference model, but in 2009 this was extended to include two intersecting measured TEM profiles. which made the site applicable for the calibration, validation, and testing of airborne TEM survey systems [Auken et al. 2011]. The aim of such reference site is to obtain the highest and most reliable data quality which is crucial for groundwater mapping in diverse geological setting [Davis et al. 2010]. This ensures that measurements collected with different, but calibrated, TEM instruments can be referred to the same geophysical response.

To this end, in 2012 the Istituto Nazionale di Geofisica e Vulcanologia (INGV) performed the calibration of a Geonics at the Lyngby reference site in Denmark. As results of the calibration, amplitude and time shift were applied to the systems which in turn allowed the Geonics instrument to strictly reproduce the Lyngby reference model. In Italy, INGV planned to set up a new reference site by the use of the previously calibrated TEM instrument. We investigated three different survey areas in the coastal Pisa Plain between the Arno River and Scolmatore Canal, Tuscany, before we turned to the area that showed better overall characteristics (Figure 1).

To establish the reference model we acquired a ground TEM sounding with the pre-calibrated Geonics TEM instrument and duly processed and inverted the collected data. The obtained reference response was then inverted using few layers models. A 5 layers resis- tivity model provide us a fit of the observed data better than 2, 3 and 4 layers models and therefore was appointed to be the TEM reference 1D resistivity model for the Italian reference site. We also checked and confirmed the recovered TEM 1D reference model by measuring at the same coincident position of the Geonics sounding with another pre-calibrated TEM instrument, the WalkTEM.

After inversion of the two collected data, both calibrated instruments provide the same model so that they can be also forced to recover exactly the same resistivity model while still fitting globally all the data within the noise level. In this paper we illustrate how we established the Italian reference and calibration site for TEM methods.

First, we show the calibration procedure adopted to calibrate the TEM instruments. Afterwards, based on the recovered reference model, we show an example of calibration of a Geonics instrument for $100 \times 100 \mathrm{~m}$ central loop configuration sounding.

\section{Method}

\subsection{Transient electromagnetic method}

The theory of the TEM method is described in several papers, as reported in the references and will not be fully discussed here [Kaufman and Keller 1983, Nabighian and Macnae 1991, Spies and Frischknecht 1991, Christiansen et al. 2009].

Time-domain electromagnetic systems operate by

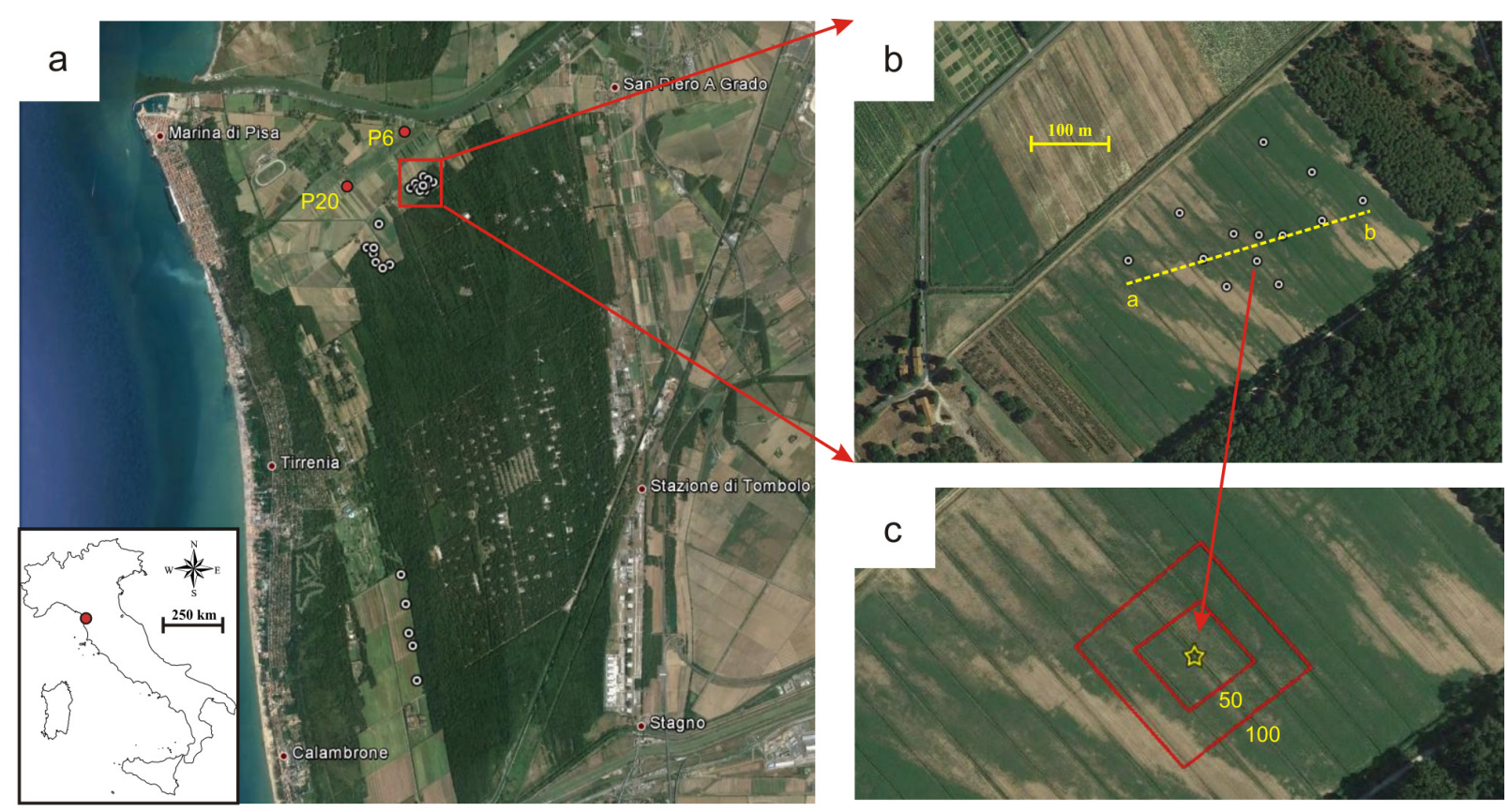

Figure 1. Location map of the Italian reference and calibration site for TEM method. (a) Red square delineates the area where the reference site is established. (b) Detailed map of the selected field area and (c) a zoom in to the reference coil position. The outer red boundaries mark the $50 \times 50 \mathrm{~m}$ and $100 \times 100 \mathrm{~m}$ loop respectively. Yellow dashed line shows the profile location illustrated in Figure 5 . Red dots locate the P20 and P6 boreholes while the white dots indicate the position of the ground-based TEM soundings performed in the entire survey area. 
abruptly turn off a current flowing through a transmitter loop, causing a sharp variation in the magnetic flux. The currents diffuse outwards and downwards into the subsurface [Nabighian 1979] and decay over time and diffuse at depth as function of ground conductivity. Those currents induce a secondary time-varying magnetic field in the medium that is measured by a receiver induction coil which is generally placed at the center of the transmitter loop.

The collected data consist of a series of variable width off-time gates. Ground conductivity affects the amplitude and the decay rate of the voltage response. Different repetition rates are implemented to handle the dynamic range of the received signal over a wide time range. Therefore, as the transient is sampled during the time-off, to achieve deeper information of the transient response, it is necessary to extend its length, by decreasing the repetition frequency. The turn-off time, which is the time interval over which the transmitter current is approximately linearly turned off, is set from the Geonics specifications (Geonics 47 Operating Manual specifications). For the Geonics system, the repetition frequencies are: ultra high $(235 \mathrm{~Hz})$ samples signals from about $7 \mu$ s (after current turn off) to $800 \mu$ s, the very high $(62.5 \mathrm{~Hz})$ samples the $35 \mu$ s to 2.8 ms time interval, the high $(25 \mathrm{~Hz})$ samples the $88 \mu$ s to $7 \mathrm{~ms}$ interval and the medium $(6.25 \mathrm{~Hz})$ covers the 350 $\mu$ s to $28 \mathrm{~ms}$ time interval.

Any changes in base frequency will affect the entire period $(\mathrm{T})$ which consists of two energizing on time period and two measurements off time period. The first measurement is normally taken at the highest repetition frequency (ultra high, $235 \mathrm{~Hz}$ ), which also collects voltage data from the very early time gates, thus providing information from the shallowest depth.

For deeper information, the signal response must be measured at very late times, the measurement period is thus extended (lower base frequency) to accurately measure the late time transient amplitudes [McNeill et al. 1984].

\subsection{Calibration procedure}

Figure 2 illustrates the calibration procedures adopted to check and calibrate all TEM systems at the Lyngby reference site for TEM methods. The calibration scheme for TEM instruments presented in this paper follows the calibration procedure described by Lavoue et al. [2010] and subsequently applied at the Danish test site [Foged et al. 2013]. In all cases, known resistivity models are used to calculate system specific forward responses and measured data are matched to the forward responses in order to define two calibration constants: amplitude and time shift factors (Figure 2).

The reference resistivity model is purely a model corresponding to the footprint of a measured TEM sounding. Therefore, calibration enables evaluation of two variables: a time shift which needs to be added (or subtracted) from each time gates for all the repetition frequencies and an amplitude shift factor by which the $\mathrm{dB} / \mathrm{dt}$ value of each gates is multiplied.

This calibration procedure assigns a time shift and an amplitude (data level) shift for individual TEM systems. The procedure is then completed when those shift factors are applied to the measured transient data until the best possible fit is achieved on the basis of a gate-bygate correlation with the reference response. After cal- a)

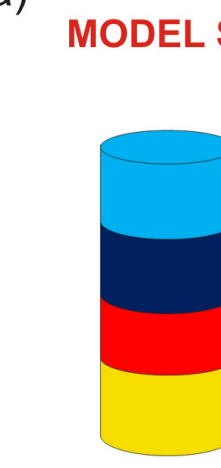

Lyngby Reference Model

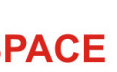

b)

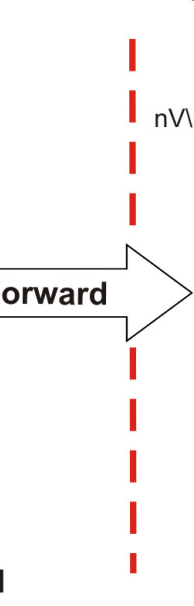

\section{DATA SPACE}

c)

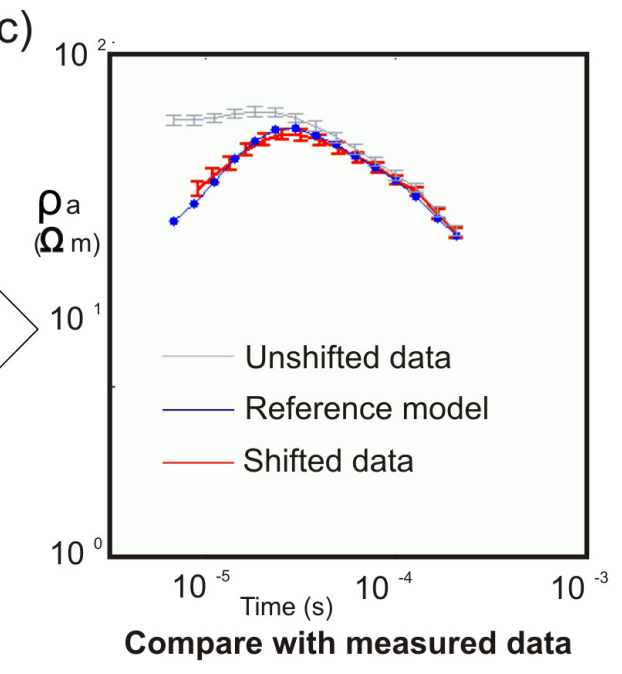

Figure 2. Simplified scheme illustrating the calibration procedure (following Foged et al. [2013]). (a) Reference 1-D resistivity model recovered from the Lyngby test site from which forward responses are calculated (b). (c) Calibration plot example for the ultra high repetition frequency. Gray curve is the raw collected TEM data. Blue curve is the reference response. Red curve is the calculated TEM response after amplitude and time shift factors correction. The TEM responses are plotted as late-time apparent resistivity for easier view. 
a)

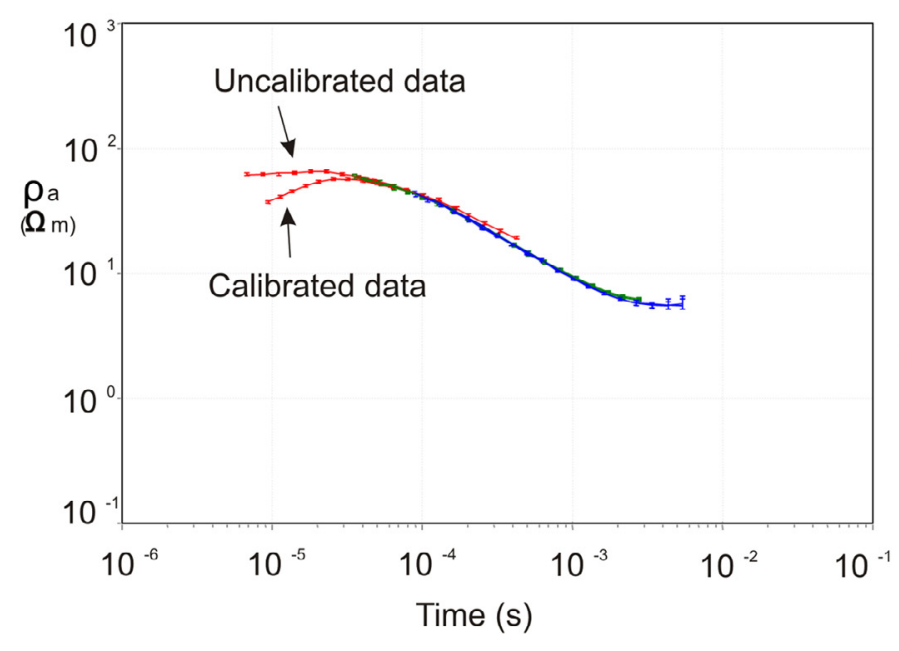

b)

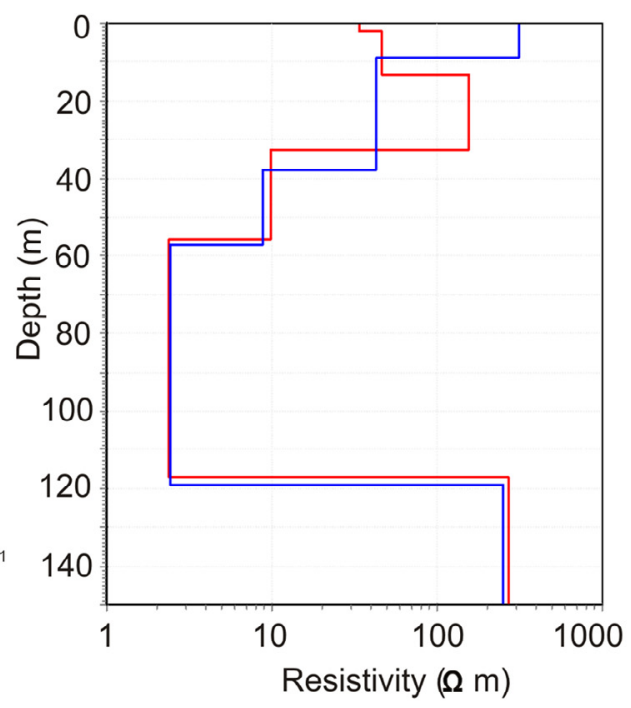

Figure 3. INGV Geonics 47 data calibrated at the Lyngby test site (Denmark). (a) Un-calibrated and calibrated forward responses. Error bars are the observed data while the colored lines are the forward responses respectively for the ultra high (UH, red curve), very high (VH, green curve) and high (Hi, blue curve) segments of the transient curve. Note that the Danish reference response match with the newly calibrated Geonics forward response. (b) 1-D resistivity models. Blue curve is the 1-D resistivity model recovered from the Geonics 47 data before the calibration. Red curve shows the 1-D reference resistivity model.

ibration, the different systems should reproduce the reference response by default within 3\% at all time gates. We minimize the least-squares misfit between the reference TEM and the measured TEM recordings by adjusting these two variables.

Figure 3 shows the results obtained after calibration of the Geonics instrument (from INGV) at the Danish test site following the calibration procedure illustrated in Figure 2. The Geonics TEM data in the example was recorded using a $50 \times 50 \mathrm{~m}$ loop with a $1 \mathrm{D}$ high frequency induction coil placed at the center of the transmitter loop. Transmitter currents of respectively $1 \mathrm{~A}$ and $3 \mathrm{~A}$ were used for the UH base frequency, and the $\mathrm{VH}$ and $\mathrm{HI}$ base frequencies.

A good fit for the early time gate for the UH measurement period was achieved by applying a time shift factor of $-0.75 \mu$ s and an amplitude factor of 0.99 as also illustrated in Figure 2c.

These apparently small shift factors give rise to the marked discrepancy observed in the apparent resistivity values for the early time gate, in turn generating incorrect very shallow model (Figure 3). Factors of 1.03 and 1.03 were applied for $\mathrm{VH}$ to $\mathrm{HI}$ repetition frequency data, respectively, in combination with time shift factors of $-1.73 \mu \mathrm{s}$ and $-1.05 \mu \mathrm{s}$. As measurement periods increase (the Rx time gates are later compared to the higher repetition frequency) the effects of minor early time shift factors generally decrease (see also Christiansen et al. [2011] for further details). This means that a time shift of for example 5-10 $\mu$ s does not have any effect on the output model when the nominal gates (to be shifted) are in the order of hundreds of $\mu$.
The effects of a given time shift become less or even negligible at later time gates. Following the calculation of calibration factors based on the test site reference response, a calibrated system transfer function (STF) was defined for use in a second inversion of the collected data.

Figure 3a shows the Geonics TEM data before and after calibration factors were applied to the STF.

Figure $3 \mathrm{~b}$ shows the Lyngby reference $1 \mathrm{D}$ resistivity model cut at $150 \mathrm{~m}$ depth [Auken et al. 2011] which is strictly confirmed by our $1 \mathrm{D}$ inversion result after the calibration (red line).

The calibrated $1 \mathrm{D}$ resistivity models are directly compared to the un-calibrated Geonics TEM 1D resistivity model recovered without using the calculated calibration factors (blu line). Note that, after calibration of the Geonics TEM system, we were able to reproduce the Lyngby reference response within 3\% at all time gates (not shown here).

\section{Results}

\subsection{The Italian reference site}

A reference and calibration site has to meet particular boundary conditions. As such, it has to be rather far from infrastructures, in an area where the general hydrogeological settings are well known, with limited expected seasonal or longer term variability of the subsurface and with limited hydrogeological spatial variability in its proximity.

For the latter, it is also important to check the repeatability of the recorded data at the selected area which 
can be done by measuring the TEM data at the same site in different period of the year.

Repeatability tests, therefore, allow to evaluate the degree of the variation in the measured data which assess mainly potential changes in hydrogeological conditions of the area.

In addition, a reference and calibration site should be located in an area not subject to foreseeable development of infrastructures, to be easily accessible and walk-able year around. Beside all this, it also has to be reasonably easy to get to by car with all the equipments and suitable for low helicopter flights.

For this purpose, we first identified from a desktop study three possible areas using available information coming from local geological map [Provincia di Pisa 2005], hydrogeological information of the area [Butteri et al. 2010, Doveri et al. 2010] together with existing borehole records. To meet the outlined conditions, we combined those ancillary data with site-specific constraints.

In general, all survey areas were located within 5 to $10 \mathrm{~km}$ from the coastline in the province of Pisa, either in farm land or in natural park. Afterwards, numerous ground based TEM measurements were collected in the selected areas using two pre-calibrated TEM instruments: the Geonics and the WalkTEM respectively. Both instruments were previously calibrated at the Lyngby test site following the Danish standard calibration procedure [Foged et al. 2013]. A total of 36 TEM soundings were conducted in the study area (Figure 1a).

We used the WalkTEM instrument with a $40 \times 40 \mathrm{~m}$ transmitter loop size and the Geonics instrument with a $50 \times 50 \mathrm{~m}$ transmitter loop size both in central loop configuration.

It soon became evident that one of the picked areas matched all the required conditions for a reference and calibration site and, therefore, we focused our efforts on this area which consisted of an open field belonging to San Rossore National Park at San Piero a Grado, Pisa (Figure 1a). We acquired several soundings, processed and modeled the data in the field in order to check whether the $1 \mathrm{D}$ condition and the TEM response against the noise level, before establishing the area as the reference and calibration site (Figure $1 \mathrm{~b}$ ).

In the selected area, a total of 13 TEM soundings were collected during February 2014 field campaign simultaneously using the Geonics 47 and the WalkTem, in a central loop configuration in order to minimize the effect of near surface - lateral resistivity variations [Adhidjaja et al. 1985].

For the Geonics 47 transmitter the turn-off time was set to $3 \mu \mathrm{s}$ as function of the loop size. This relatively short turn-off time allows for a proper descrip- tion of the resistivity properties of the uppermost parts of the subsurface. The decay of the secondary magnetic field, recorded by the receiver coil, is sampled over three segments: ultra high, very high and high repetitions rates. For each segment, transient decay is recorded at 20 time gates.

The optimum gain setting was set to be the highest gain value that can be implemented, without causing saturation or non-linearity of the readings, due to excessive effect of background noise.

Input current is set to be $1 \mathrm{~A}$ for the ultra high and $3 \mathrm{~A}$ for the very high and high repetition rates respectively. Since we selected an area fairly far from humaninfrastructures, we avoided local sources of significant electromagnetic noise, coming from power line, pipes, fences affecting the collected data [Denielsen et al. 2003]. Nevertheless, background noise data was recorded in order to check and assess late time noise levels in the measured data, while several records were acquired for each set of measurements in order to improve the signal to noise ratio [Munkholm and Auken 1996].

Figure 4 shows an example of a measured transient data for a $50 \times 50 \mathrm{~m}$ loop using the calibrated Geonics 47. The observed high signal to-noise ratio, is interpreted to be a result of the combined effects of the very few cultural noise sources in the area where measurements have been conducted and the response of a shallow geological feature with high electrical conductivity.

The data was then duly processed and inverted to

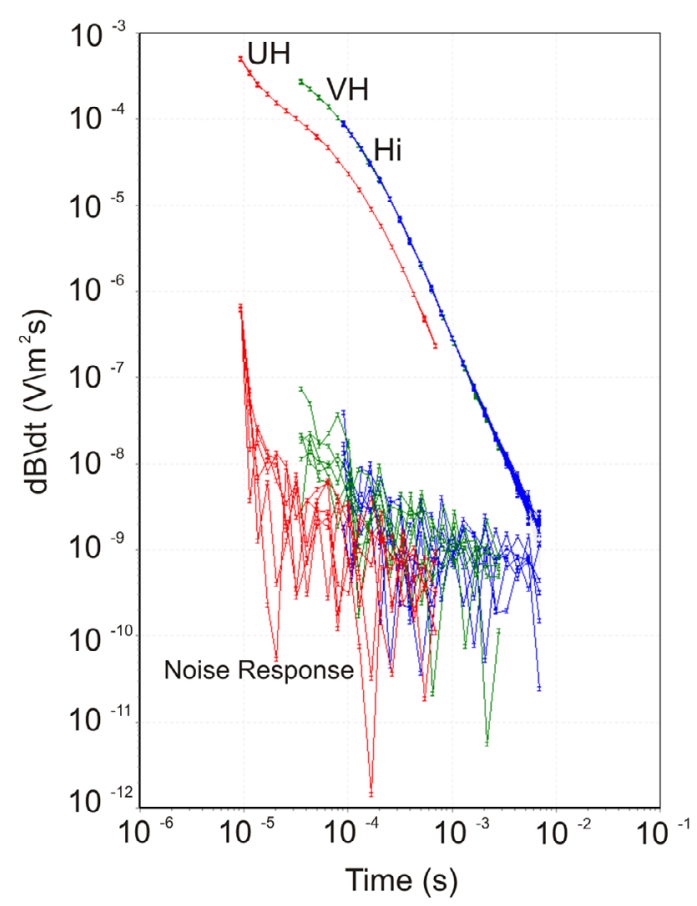

Figure 4. Signal and noise responses measured for the ultra high $(\mathrm{UH})$, very high $(\mathrm{VH})$, and high $(\mathrm{HI})$ segments. The color used for the TEM response, in a given time segment, corresponds to that for noise values measured in the same time segment. 


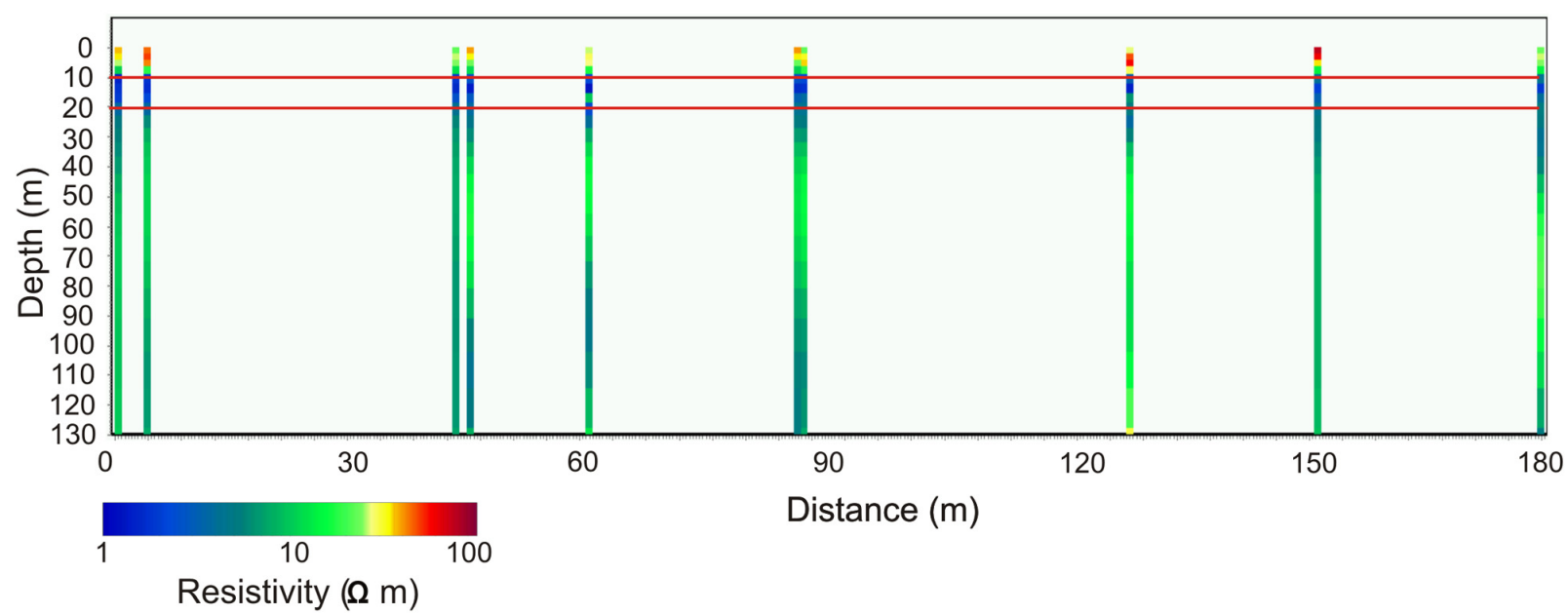

Figure 5. WSW-ENE resistivity section as illustrated in Figure 1b. Smooth inversion results calculated for each soundings are plotted in the profile section. Top and bottom red lines highlight a $10 \mathrm{~m}$ thick clay layer throughout the profile.

smooth and blocky models.

Initial processing of the TEM data included editing, averaging and definition of a-priori uncertainties. This was performed by means of the SiTEM software.

The TEM data were then inverted in order to define 1-D resistivity models of the subsurface using the SEMDI program. Both the SiTEM and SEMDI software were developed by the Hydrogeophysics Group, Aarhus University [Auken and Nebel 2001]. The iterative least squares algorithm used to achieve inversion (modeling the complete system response) requires a starting model. One of the major advantages of using least squares-based inverse methods is that they provide an assessment of model resolution [Auken et al. 2002].

The model resolution estimates for individual model parameters are presented in relative standard deviation numbers. An ideal model parameter exhibits a resolution value of 1.00 , while a resolution value of 1.05 indicates an estimated 5\% uncertainty of the relevant model parameter.

Generally low resistivity layers can be properly resolved using the TEM methods, while high resistivity layers are poorly resolved. An a-priori starting model is defined for each inversion as a homogeneous half space.

Figure 5 shows a NE-SW oriented profile section which intersect 10 TEM soundings from a total of 13 soundings acquired in the survey area (Figure 1b). Data were inverted using a 29 layers starting model each having a fixed thickness but free resistivity (soft constrains).

From the recovered smooth models we observe fairly $1 \mathrm{D}$ lateral condition of the main resistivity contrast throughout the profile. A moderately resistive layer $(30 \Omega \mathrm{m}$ ), covers the first $10 \mathrm{~m}$ from the surface and lies above a thin, approximately $10 \mathrm{~m}$ thick, low resistive layer $(1.5 \Omega \mathrm{m})$.

Below this layer, 1D models show homogeneous resistivity values of $10 \Omega \mathrm{m}$ up to a depth of $90 \mathrm{~m}$ which also correspond to the averaged depth of investigation (DOI) [Christiansen and Auken 2012].

The receiver induction coil position for the final reference sounding was chosen in the center of the survey area, where the lateral variability of the subsurface resistivity was lower. On that position, we therefore acquired two TEM soundings with a $50 \times 50 \mathrm{~m}$ and $40 \times$ $40 \mathrm{~m}$ central loop respectively using the Geonics and the WalkTEM previously calibrated at the Danish test site. Figure 6 shows the reference forward responses plotted as late-time apparent resistivity curves and the $1 \mathrm{D}$ resistivity models recovered after data inversion. Despite the different footprint $(50 \times 50 \mathrm{~m}$ against $40 \times 40$ $\mathrm{m}$ loop size), they show very similar models, so that they can be jointly inverted to recover exactly the same resistivity model while still fitting globally the observed data within the noise level (Figure 7).

A five layered model is sufficient to explain the recorded data. The reference model parameters with model uncertainty (expressed as standard deviation, STD) estimated from the 1D inversion calculation are shown in Table 1. The shallowest layer, despite its moderate resistivity, shows very low uncertainties due to its enhanced signal response compared to the background noise. Conversely, the deepest layer of the model is clearly unresolved because of its high resistivity and depth.

Model parameter analysis shows also very low uncertainties for layers 2 and 3 of the recovered resistivity model which reflect the higher capability of the method to better resolve conductive targets. Thicknesses and depth to layers standard deviations clearly increase for layers 3 and 4 due to progressively greater depth although the fourth layer shows up as very low resistivity response.

We note a good match comparing the obtained resistivity model with stratigraphic information derived 
a)
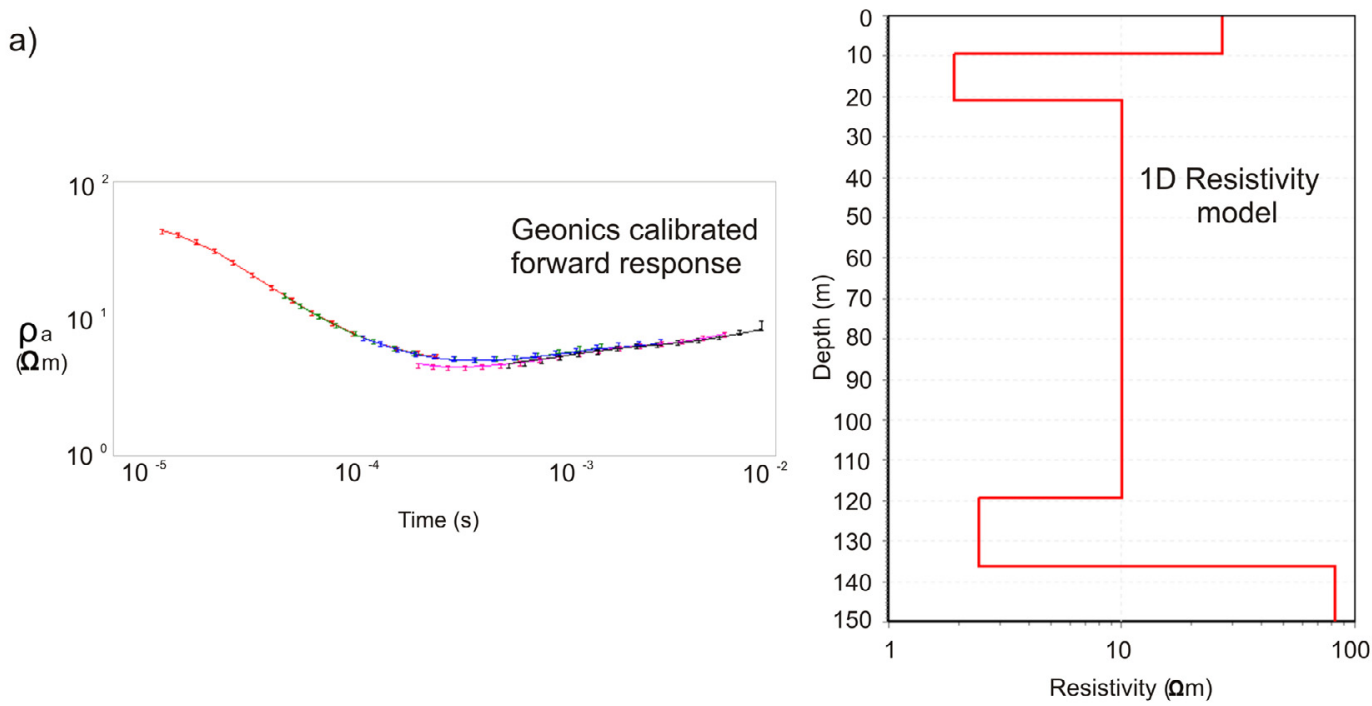

b)
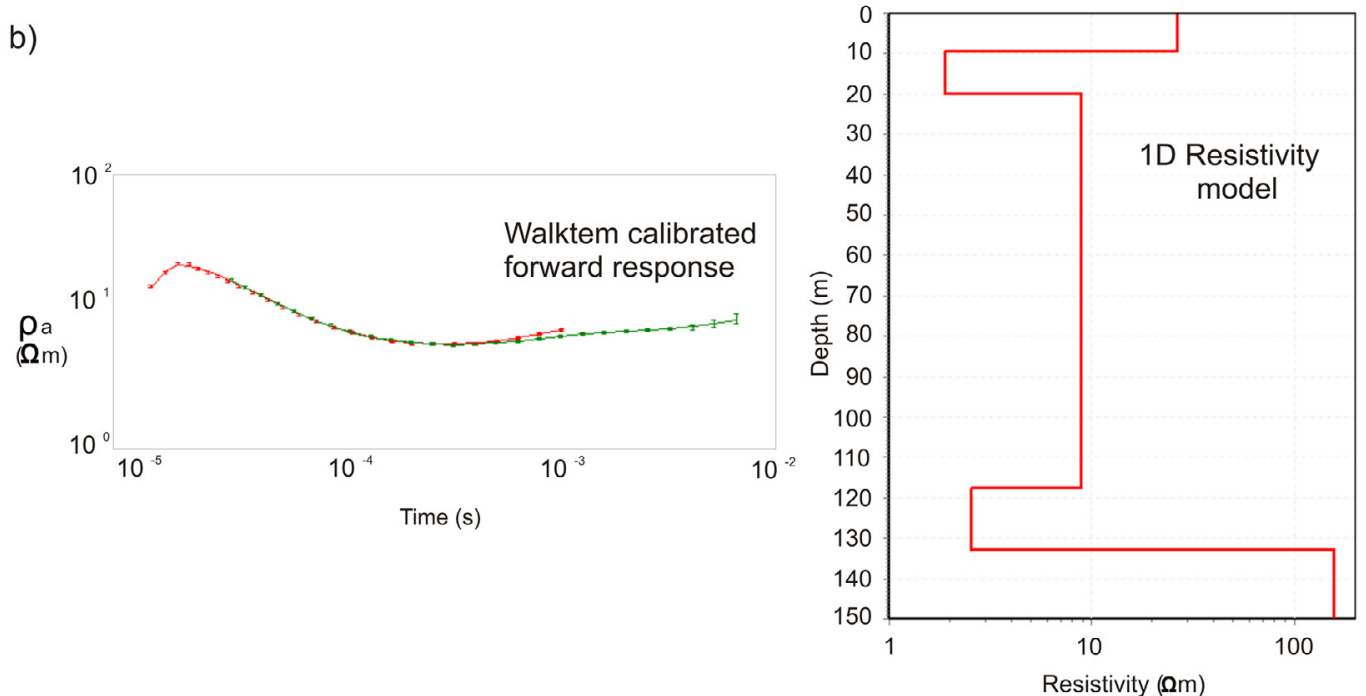

Figure 6. Field data (error bar), forward responses (solid line) and recovered 1-D resistivity models for the two calibrated (a) Geonics 47 and (b) WalkTEM instruments obtained at the Italian reference and calibration site for coincident coil position from a $50 \times 50 \mathrm{~m}$ and $40 \times 40 \mathrm{~m}$ loop size respectively.

a)

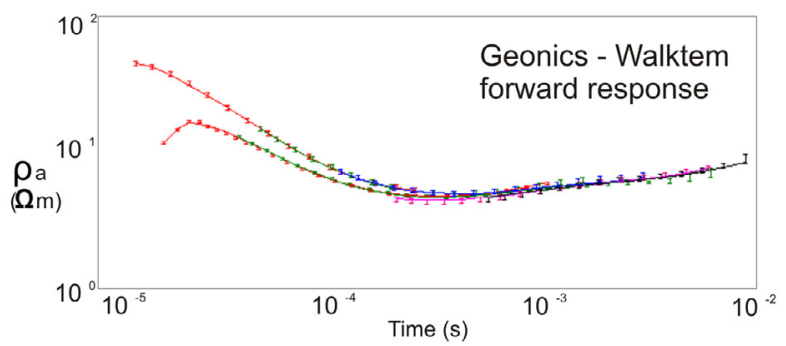

Figure 7. Joint inversion result of the collected Geonics 47 and WalkTEM data at the Italian reference and calibration site. a) Forward response (solid line) fit the observed data (error bars) within the noise level (set to 3\% by default). b) Recovered 1-D resistivity model. b)

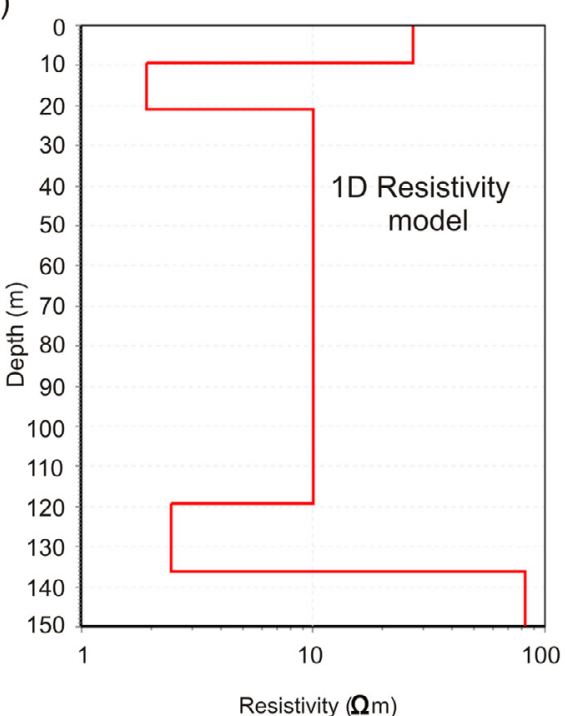


SAPIA ET AL.

\begin{tabular}{|c|c|c|c|c|c|c|}
\hline & $\begin{array}{l}\text { Resistivity } \\
\quad(\mathbf{m})\end{array}$ & $\begin{array}{l}\text { Thickness } \\
(\mathbf{m})\end{array}$ & $\begin{array}{c}\text { Depth } \\
(\mathbf{m})\end{array}$ & $\begin{array}{c}\text { Resistivity } \\
\text { STD }\end{array}$ & $\begin{array}{c}\text { Thickness } \\
\text { STD }\end{array}$ & $\begin{array}{l}\text { Depth } \\
\text { STD }\end{array}$ \\
\hline Layer 1 & 24.8 & 9.9 & 9.9 & 1.05 & 1.02 & 1.02 \\
\hline Layer 2 & 1.8 & 10.3 & 20.2 & 1.05 & 1.10 & 1.04 \\
\hline Layer 3 & 11.8 & 97.5 & 117.7 & 1.10 & 1.17 & 1.21 \\
\hline Layer 4 & 2.8 & 23 & 130.7 & 1.16 & 1.33 & 1.25 \\
\hline Layer 5 & 84.8 & --- & --- & unresolved & unresolved & unresolved \\
\hline
\end{tabular}

Table 1. Model parameter analysis for the 5 layer $50 \times 50 \mathrm{~m}$ loop reference model at the San Rossore reference site. The model parameter uncertainties are stated as STD-factors [Auken et al. 2005]. STD below 1.2 indicates well resolved parameters while STD above 2 corresponds to unresolved parameters.

from two nearby boreholes (Figure 8; see Figure 1a for borehole location).

In particular, the 1D inversion result shows a shallower moderately resistive layer, $10 \mathrm{~m}$ thick, which corresponds to the sand and silty-clay materials sampled in the borehole. Then, the resistivity model indicates the presence of a thin (10 $\mathrm{m}$ thick) low resistive layer (1.8 $\Omega \mathrm{m}$ ) which can be attributable to the response of a clay and silt sediments. A uniform resistivity of about 10 $\Omega \mathrm{m}$ is observed up to a depth of $115 \mathrm{~m}$ which correspond to the top of a lower resistive layer, approximately $15 \mathrm{~m}$ thick, lying above a more resistive layer at the bottom. Direct geological correlation with the deep resistive layer is hampered since this layer lies at a depth greater than the maximum penetration drilled from the borehole. In fact, the P6 borehole (Figure 8, left side)

a)

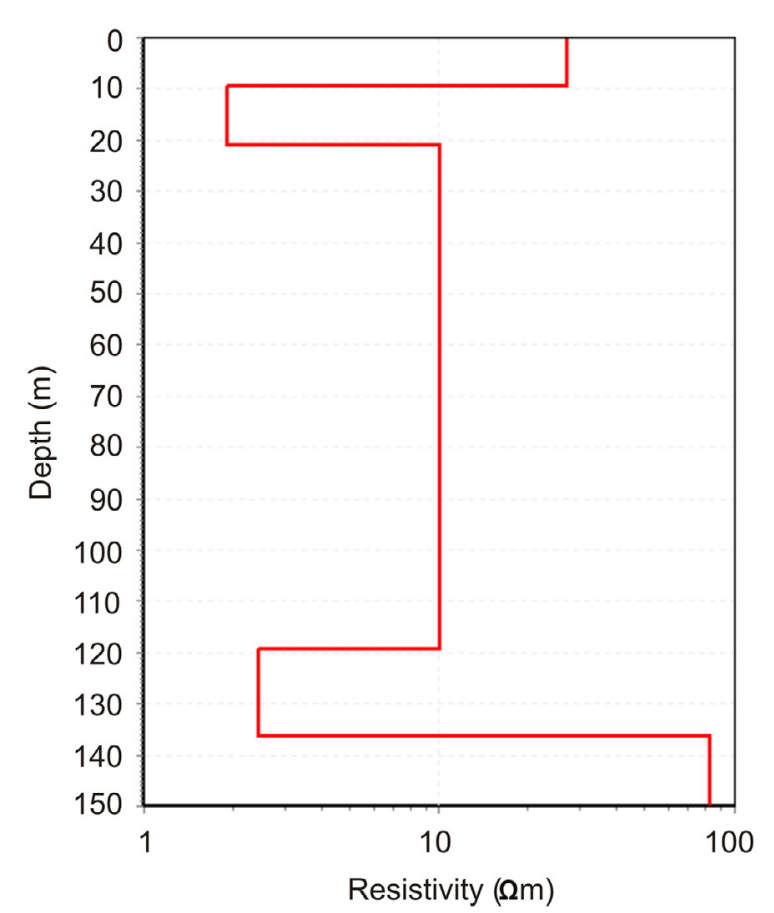

sampled few meters of gravel mixed to sand and silt materials at a depth of about $117 \mathrm{~m}$ which corresponds to the top of the deep conductor in the recovered 1D resistivity model (Figure 8, right side), before drilling stopped at about $125 \mathrm{~m}$ depth.

\subsection{Calibration of the Geonics 47 and 57 for $100 \times 100$} $m$ transmitter loop

The 5 layers reference model was subsequently used to calibrate the Geonics 47 and 57 for a $100 \times 100$ central loop sounding at the same location using the presented calibration scheme. Therefore, we placed the $100 \times 100 \mathrm{~m}$ loop in a way that the induction receiver coil exactly holds the same reference sounding position (Figure 1c).

By doing this, we avoided discrepancies in the out-

b)

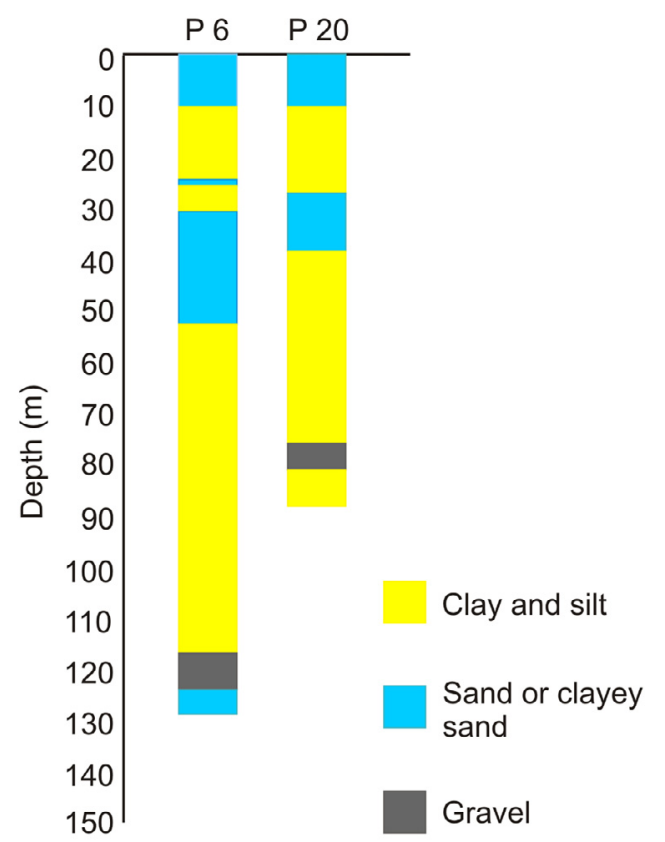

Figure 8. (a) 1-D reference resistivity model of the Italian reference site. Data inversion recovered a 5 layers resistivity model which provides us the best fit of the observed data. (b) Simplified representation of the boreholes stratigraphy (adapted from Butteri et al. [2010]). 
put resistivity model parameters caused by variations in the coil position.

A Geonics forward response of the reference model was calculated, using the Geonics system transfer function. The calibration was then completed by applying small uniform level and time shifts to the measured data-curve to obtain the best possible fit with the reference response. Discrepancies between the reference response and observed data show up as a mismatch mainly at early times. Figure 9 shows an example of the gate by gate calibration procedure and the obtained shift factors for the UH repetition frequency.

To fit the early time gates as regards the reference response for the UH measurement period, a time shift factor of $1.1 \mu \mathrm{s}$ and an amplitude shift factor of 1.01 was calculated. Factors of 1.04, 1.07 and 1.03 were applied for $\mathrm{VH}, \mathrm{HI}$ to $\mathrm{MD}$ repetition frequency data, respectively, together with a time shift factors of $-6.68 \mu \mathrm{s}$, $6.08 \mu$ s and $13.47 \mu$ s (Table 2).

Following the calculation of calibration factors based on the site reference response, a calibrated system transfer function (STF) was defined and then adopted for a second inversion of the collected data. Data were obtained from approximately $9 \mu$ s to $20 \mathrm{~ms}$.

We inverted the data with a least-squares inversion algorithm using the Semdi programme. Figure 10 shows the recovered $1 \mathrm{D}$ resistivity model for the calibrated $100 \times 100 \mathrm{~m}$ loop sounding.

\section{Discussion}

The concept of the Italian reference and calibration site rests on the assumption that we can use calibrated TEM instruments to check other TEM instruments and calibrate them in Italy. Therefore, TEM equipments from other organizations can be hosted for the same purpose.

\section{a)}

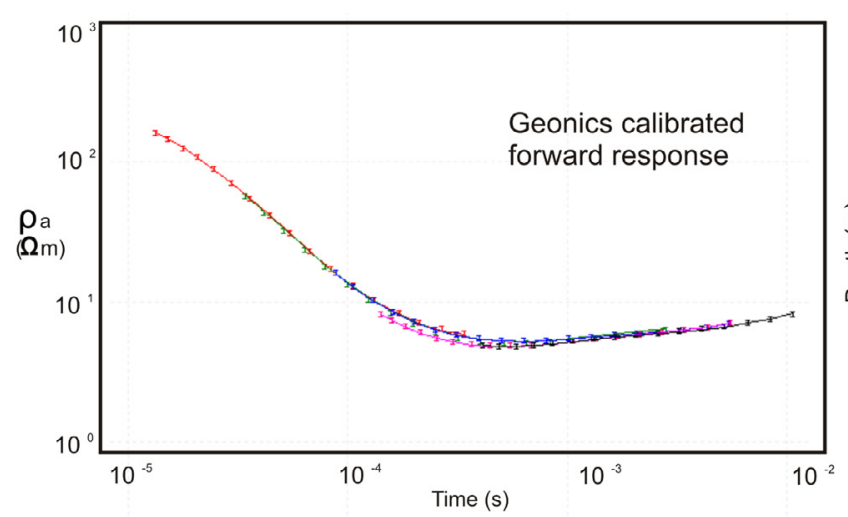

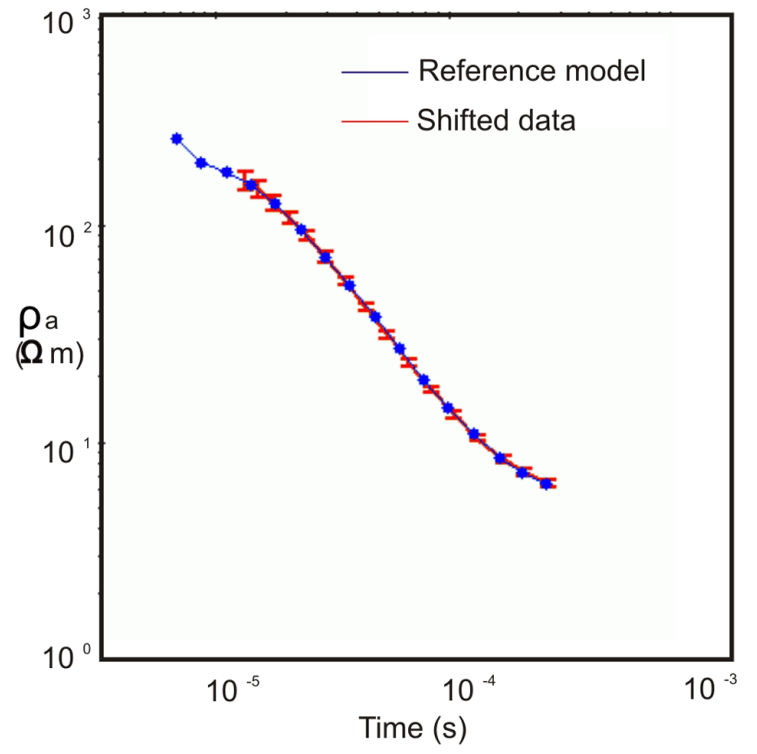

Figure 9. Comparison between the reference response (blue curve) and the shifted (red curve) data for the ultra high (UH) segment after calibration for a $100 \times 100 \mathrm{~m}$ loop.

\begin{tabular}{lcc}
$\begin{array}{l}\text { Repetition } \\
\text { frequencies } \\
\text { (47 transmitter) }\end{array}$ & $\begin{array}{c}\text { Time shift } \\
\text { factors }\end{array}$ & $\begin{array}{c}\text { Amplitude shift } \\
\text { factors }\end{array}$ \\
\hline Ultra high & $1.10 \mu \mathrm{s}$ & 1.014 \\
Very high & $-6.58 \mu \mathrm{s}$ & 1.049 \\
High & $-5.5 \mu \mathrm{s}$ & 1.077 \\
\hline
\end{tabular}

Repetition frequencies ( 57 transmitter)

\begin{tabular}{lcc}
\hline High & $6.08 \mu \mathrm{s}$ & 1.038 \\
Middle & $13.47 \mu \mathrm{s}$ & 1.039 \\
\hline
\end{tabular}

Table 2. Time and amplitude shift factors calculated for the INGV Geonics 47 and 57 for a $100 \times 100 \mathrm{~m}$ transmitter loop.

b)

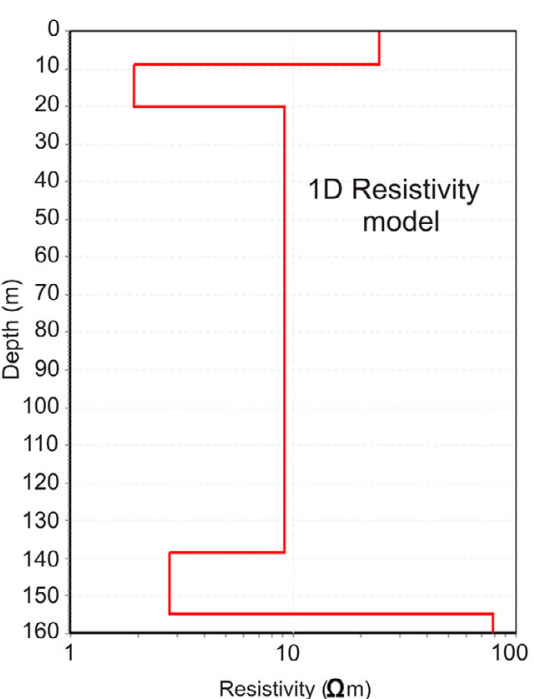

Figure 10. (a) Forward response of the calibrated $100 \times 100 \mathrm{~m}$ loop data plotted as late-time apparent resistivity. (b) Recovered 5 layers 1-D resistivity model after data inversion. 
a)

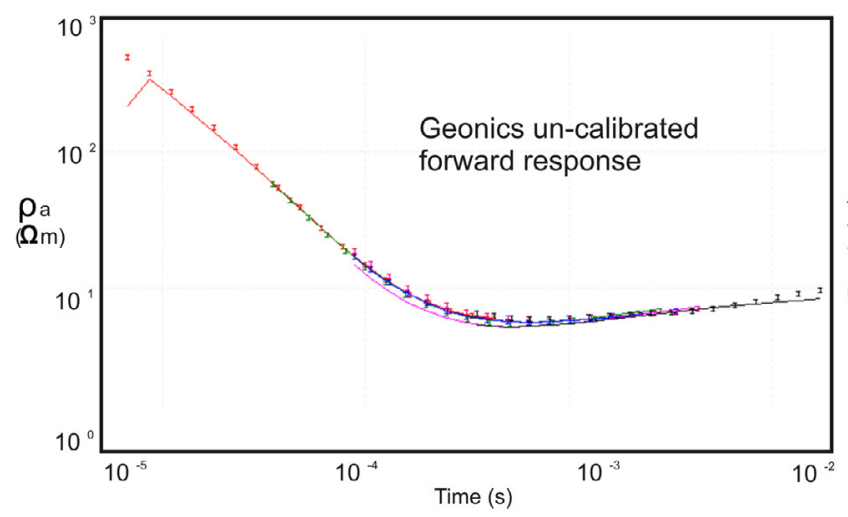

b)

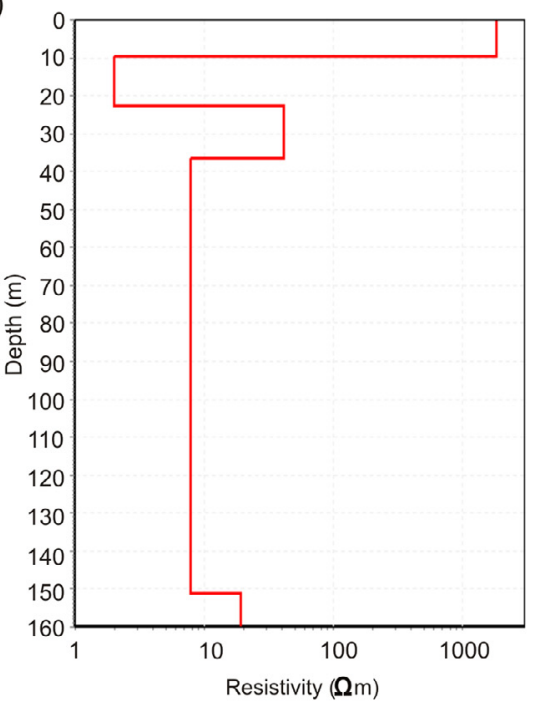

Figure 11. Un-calibrated $100 \times 100$ loop response obtained without amplitude and time shift factors correction. (a) Forward response (solid line) can not fit the observed data (error bars). (b) Recovered 1-D resistivity model

In order to achieve this purpose, it is mandatory to find suitable site, duly acquire data with calibrated TEM instruments, process and model the data using state of the art procedures that avoid artifacts in the modeling.

Repeatability test provides us very low degree of variability $(<2 \%)$ between the observed data acquired during two different period of the year, February and November respectively, for the same coil position (not shown here). As such, changes in the ground resistivity due to seasonal hydrogeological variations in the survey area could be ruled out.

On the quest for a suitable site in Italy, we drew on the vast and well documented experience from the Danish test site. The reference site is placed at a location with a relatively high TEM response compared to the noise level as well as relatively homogenous lateral conditions.

The results presented herein are a direct confirmation of the applicability and robustness of this overall approach and of the accuracy of the newborn reference site of San Piero a Grado (Pisa).

The reference model is purely a resistivity model which provides information corresponding to the footprint of a TEM sounding. We therefore have obtained a calibrated 1D resistivity model that can be used as reference to check the performance of other TEM instruments of known technical characteristics. The concept is similar to that applied routinely to calibrate instruments which are checked against standards (e.g., the specimen of the metre or kilograms established in 1790 during the French Revolution) or their derivate. The only difference in this procedure is that we cannot compare the reading of different instruments only in the data space, because different instruments have different system transfer function and therefore they can "see" equiva- lent Earth responses through different lenses. We therefore need to go also by a common $1 \mathrm{D}$ ground resistivity model and calibrate the system in the model space.

At first glance the lack of ground-truth against the recovered $1 \mathrm{D}$ resistivity model might seem a limitation of the proposed reference site. However, the aim of such reference site is to ensures that measurements can be referred to the same geophysical ground response regardless the geological setting. Of course, the reference model should not be in disagreement with the general accepted geological and hydrogeological settings of the area.

The shift factors of the data gates are applied to force general equality (within noise levels) between the modeled and observed TEM responses. Notice that, if we model the data of the i.e. Geonics instrument over the Italian reference site without applying the Italian calibration factors, the results obtained would be in significant disagreement. Figure 11 shows the results of the un-calibrated $100 \times 100$ loop response. We observe high misfit between the forward response (solid line) and the observed data (error bars) at the early and middle time gates of the transient curve (Figure 11a). This results in an erroneous output $1 \mathrm{D}$ resistivity model with high resistivity at the very near surface which significantly differ from the reference $1 \mathrm{D}$ resistivity model (Figure 11 compared to Figure 10).

The suggested approach can also be applied to calibrate any airborne EM dataset before a survey occurs. The importance to have a calibrated system lies directly onto the possibility to gather accurate data to be further interpreted to obtain reliable geological and hydrogeological models. This is a pragmatic approach. In fact, when calculating the time shift and amplitude shift factors with the above mentioned procedure, we do not attempt to characterize the source of the timing errors 
within the various instruments tested. Although the present state of the art suggests that those calculated factors shift are assumed to come from different system related issues like, transmitter waveform, transmitterreceiver timing, low pass filters [Davis and Macnae 2008, Christiansen et al. 2011], they substantially include all system's inaccuracies which may affect the transient response of the Earth. Regardless of the source of these issues, the application of the correction factors to the collected data set yields the resistivity model of the terrain of the reference site.

\section{Conclusions}

This paper provides a detailed description of the reference and calibration site established in Italy which we propose as support for the calibration of any ground and airborne TEM systems that will operate in Italy, following the experience maturated in Denmark.

We prove that it is possible to use a calibrated instruments to set up new reference sites in other parts of the world. We claim that, like Lyngby in Denmark, the reference and calibration site we set out to establish in Italy, is strictly a site for testing and calibrating TEM instruments and not a site to calibrate geophysics against geology or TEM against other geophysical data.

The procedure presented herein is a pragmatic and functional solution that will ensure consistency across TEM derived models (not just resistivity models, but potentially also geological and hydrogeological) obtained over the years by different contractors with different systems.

We argue it will play an important role in largescale groundwater and geological mapping that we hope will take place in the near future in our country, using also TEM surveys.

Acknowledgements. The project of the Italian reference site for TEM methods has been carried out thanks to the cooperation of several partners. First of all we kindly thank the San Rossore Park authorities which supported the study and provided us a area to establish the reference site. We are also grateful to Marco Doveri (Istituto di Geoscienze e Georisorse, CNR of Pisa) and Roberto Giannecchini (Dipartimento di Scienze della Terra, University of Pisa) for fruitful discussion on the geological and hydrogeological setting of the area together with their technical and logistical support in data acquisition. Thanks to Domenico di Massa (University of Naples), Fabio Giannino (Geophysical Consultant), Sandra Trifirò, Matteo Butteri and Camilla Sørensen (University of Adelaide) for their great support in the field work and for the Walktem instrumentation. We also thank Prof. Giovanni Santarato and an anonymous reviewer for their constructive reviews of our manuscript.

\section{References}

Adhidjaja, J.I., G.W. Hohmann and M.L. Oristaglio (1985). Two dimensional transient electromagnetic responses, Geophysics, 50, 2849-2861.
Auken, E., and L. Nebel (2001). Getting started with SiTEM and SEMDI, Hydrogeophysics Gruup, University of Aarhus, Denmark, 1-22.

Auken, E., L. Nebel, K. Sørensen, M. Breiner, L Pellerin and N.B. Christensen (2002). EMMA - a geophysical training and education tool for electromagnetic modeling and analysis, J. Environ. Eng. Geophys., 7, 57-68.

Auken, E., A.V. Christiansen, B.H. Jacobsen, N. Foged and K.I. Sørensen (2005). Piecewise 1D laterally constrained inversion of resistivity data, Geophys. Prosp., 53, 497-506.

Auken, E., A.V. Christiansen, N. Foged, C. Schamper, J. Pedersen and K.I. Sørensen (2011). Refinement of the national TEM reference model at Lyngby, Department of Geoscience, Aarhus University; www. hgg.geo .au.dk/rapporter/Refinement_TEM_Ref_ Model_Lyngby.pdf.

Butteri, M., M. Doveri, R. Giannecchini and P. Gattai (2010). Hydrogeologic-hydrogeochemical multidisciplinary study of the confined gravelly aquifer in the coastal Pisan Plain between the Arno River and Scolmatore Canal (Tuscany), Mem. Descr. Carta Geol. d'It., XC, 51-66.

Christiansen, A.V., E. Auken and K. Sørensen (2009). The transient electromagnetic method, In: Groundwater geophysics, Springer Berlin Heidelberg, 179226.

Christiansen, A.V., E. Auken and A. Viezzoli (2011). Quantification of modeling errors in airborne TEM caused by inaccurate system description, Geophysics, 76, 43-52.

Christiansen, A.V., and E. Auken (2012). A global measure for depth of investigation, Geophysics, 77, 171177.

Danielsen, J.E., E. Auken, F. Jørgensen, V.H. Søndergaard and K.I. Sørensen (2003). The application of the transient electromagnetic method in hydrogeophysical surveys, J. App. Geophys., 53, 181-198.

Davis, A., and J. Macnae (2008). Measuring AEM waveforms with a ground loop, Geophysics, 73, 213-222.

Davis, A., Y. Ley-Cooper and C. Kirkegaard (2010). SkyTEM system calibration: Two systems, one dataset, In: Proceeding: 21st Geophysical Conference, ASEG, 1-4.

Doveri, M., R. Giannecchini and M. Butteri (2010). Seawater intrusion in the Versiliese Pisan coastal aquifer system (northwestern Tuscany): results from a hydrogeologic - hydrogeochemical study, In: Proceeding: 21st Salt Water Intrusion Meeting, Azores, Portugal, 150-153.

Foged, N., E. Auken, A.V. Christiansen and K.I. Sørensen (2013). Test site calibration and validation of air- 
borne and ground based TEM systems, Geophysics, 78, 95-106.

Kaufman, A.A., and G.V. Keller (1983). Frequency and transient sounding, Elsevier, $685 \mathrm{p}$.

Lavoue, F., J. Van Der Kruk, J. Rings, F. Andre, D. Moghadas, J.A. Huisman, S. Lambot, L. Weihermüller, J. Vanderborght and H. Vereecken (2010). Electromagnetic induction calibration using apparent electrical conductivity modelling based on electrical resistivity tomography, Near Surf. Geophys., 8, 553-561.

Liu, G. (1998). Effect of transmitter current waveform on airborne TEM response, Expl. Geophys., 29, 3541.

McNeill, J.D., M. Bosnar and G.M. Levy (1984). Application of simple loop to the interpretation of transient electromagnetic surveys in a resistive environment, Geonics Limited, Technical note TN-12, 1-13.

Munkholm, M.S., and E. Auken (1996). Electromagnetic noise contamination on transient electromagnetic soundings in culturally disturbed environments, J. Environ. Eng. Geophys., 1, 119-127.

Nabighian, M.N. (1979). Quasi-static transient response of a conducting half-space. An approximation representation, Geophysics, 44, 1700-1705.

Nabighian, M.N., and J.C. Macnae (1991). Appendix A: TEM systems, In Electromagnetic methods in applied geophysics, Nabighian, M.N., Ed., II, 479-483.

Nielsen, L., N.O. Jørgensen and P. Gelting (2007). Mapping of the freshwater lens in a coastal aquifer on the Keta Barrier (Ghana) by transient electromagnetic soundings, J. App. Geophys., 62, 1-15.

Podgorski, J.E., E. Auken, C. Schamper, A.V. Christiansen, T. Kalscheuer and A.G. Green (2013). Processing and inversion of commercial helicopter time-domain electromagnetic data for environmental assessments and geologic and hydrologic mapping, Geophysics, 78, 149-159.

Provincia di Pisa (2005). La Geologia della Provincia di Pisa - Cartografia, Geositi e Banche Dati. Provincia di Pisa, Area Governo del Territorio-Servizio Difesa del Suolo.

Santilano, A., A. Manzella, A. Donato, D. Montanari, G. Gola, E. Di Sipio, E. Destro, A. Giaretta, A. Galgaro, G. Teza, A. Viezzoli and A. Menghini (2015). Shallow Geothermal Exploration by Means of SkyTEM Electrical Resistivity Data: An Application in Sicily (Italy), Eng. Geol. Soc. Territory, 1, 363-367.

Spies, B.R., and F.C. Frischknecht (1991). Electromagnetic sounding, In: M.N. Nabighian (ed.), Electromagnetic Methods in Applied Geophysics - Applications, Society of Exploration Geophysicists, 2, 285425.
Corresponding author: Vincenzo Sapia, Istituto Nazionale di Geofisica e Vulcanologia, Rome, Italy; email: vincenzo.sapia@ingv.it.

(C) 2015 by the Istituto Nazionale di Geofisica e Vulcanologia. All rights reserved. 